\title{
La réception du cinéma à Taiwan sous domination coloniale japonaise : une « assimilation par confrontation »
}

植民地期台湾における映画受容の「臨場的土着化」

Film Reception in Taiwan under Japanese Rule, or "Assimilation by

Confrontation"

\section{Mamie Misawa}

Traducteur : Arnaud Nanta et Laurent Nespoulous

\section{OpenEdition}

\section{Journals}

Édition électronique

URL : https://journals.openedition.org/ebisu/1923

DOI : 10.4000/ebisu.1923

ISSN : 2189-1893

Éditeur

Institut français de recherche sur le Japon à la Maison franco-japonaise (UMIFRE 19 MEAE-CNRS)

Édition imprimée

Date de publication : 10 décembre 2016

Pagination : 203-235

ISSN : 1340-3656

\section{Référence électronique}

Mamie Misawa, «La réception du cinéma à Taiwan sous domination coloniale japonaise : une " assimilation par confrontation » », Ebisu [En ligne], 53 | 2016, mis en ligne le 10 décembre 2016, consulté le 08 novembre 2021. URL : http://journals.openedition.org/ebisu/1923 ; DOI : https:// doi.org/10.4000/ebisu. 1923 


\section{La réception du cinéma à Taiwan sous domination coloniale japonaise}

Une " assimilation par confrontation »

Misawa Mamie

Film Reception in Taiwan under Japanese Rule, or "Assimilation by Confrontation"

Misawa Mamie

\begin{abstract}
\ Mots-clés : Taiwan, Japon, colonisation, cinéma, assimilation.
\end{abstract}

L'auteure: Misawa Mamie est historienne, professeure à l'université Nihon. Elle est spécialiste de l'histoire du cinéma de langue chinoise et particulièrement à Taiwan, ainsi que des relations entre colonisation, nationalisme et médias.

Résumé : La question de la réception des œuvres cinématographiques, avant tout japonaises, puis chinoises, américaines et européennes, par les populations de Taiwan entre 1895 et 1945 se pose avec acuité du fait de la situation coloniale de l'île durant cette période. Le présent article tente de montrer comment une autre culture que celle du cinéma des États-nations apparut à Taiwan durant la période coloniale, culture qui ne rejeta pas l'altérité mais l'intégra au travers d'un processus original que nous appelons « assimilation par confrontation". Ce processus entraîna des événements cinématographiques où le colonisé se réappropria le cinéma. Il s'agit donc aussi de réfléchir aux raisons pour lesquelles on constate une telle différence dans la réception des œuvres en situation coloniale. 
マキーワード

台湾、日本、植民地、映画、土着化

\section{著者}

三澤真美恵は日本大学教授、歴史学者。台湾 を中心とした華語圈の映画史を専門とし、植 民地主義やナショナリズムとメディアとの関 係を研究している。

\section{要旨}

台湾は 1895 年から 1945 年にかけて日本に よる植民地統治下にあり、島内での映画製作
は産業化していなかつたが、日本製映画を筆 頭に、中国製、米国製、ヨーロッパ製など多 様な映画が流通していた。本稿では、「臨場的 土着化」という独特なプロセスを通じて、植 民地期台湾人の映画受容が、帝国日本という

「他者」が提供する映画（コンテンッ）を排 除することなく、いかにして「我々」の映画 （イベント）を創出したのかを論じる。これは、 言い換えるならば、国民国家的な「映画受容」 とは異なる、植民地的な「映画受容」が、い かにその独自性を獲得することが可能だった か、を論じることでもある。
\ Keywords: Taiwan, Japan, Colonisation, Cinema, Assimilation.

The Author: Misawa Mamie is a historian and professor at Nihon University. She specialises in the history of Chinese-language cinema, in particular in Taiwan. Other research interests include the links between colonisation, nationalism and media.

Abstract: The question of how filmsmainly Japanese but also Chinese, American and European - were received by the people of Taiwan between 1895 and 1945 is of particular interest due to the island being under colonial rule during this period. This paper seeks to illustrate how, during the colonial period, the Taiwanese did not reject otherness but rather assimilated it by way of a unique process I term "assimilation by confrontation". This process saw the appearance of cinematographic events in which the colonised population appropriated such foreign productions. To rephrase, film reception in Taiwan during the colonial period was not that of a nation-state but that of a colonised territory. I aim to explore how such a difference in film reception in a colonial context was possible. 


\title{
La réception du cinéma à Taiwan sous domination coloniale japonaise
}

\author{
Une « assimilation par confrontation »
}

MISAWA Mamie 三澤真美恵*

L'année où les frères Lumière inventaient le cinématographe, Taiwan était annexé par le Japon et cessait, suivant les résolutions de la guerre sinojaponaise (1894-1895), d'être une possession de l'empire Qing. La diffusion du cinéma dans l'île débuta donc en situation coloniale. La présente contribution a pour objectif de saisir les mécanismes de la réception des productions cinématographiques dans la société taiwanaise alors sous le joug des Japonais, à l'époque des grands processus qui, bon an mal an, étaient en train de faire émerger, en Chine et au Japon, une première conscience nationale.

La période d'introduction des premiers films à Taiwan est encore discutée mais, d'après les recherches les plus récentes, elle commencerait en 1898 (Hong Yawen 2000 : 67). Si nous prenons pour critère la formation d'un marché du film et ses transformations à mesure de sa rationalisation, il devient possible de diviser la progression de la diffusion du cinéma à Taiwan en trois phases (Misawa 2010 : 39-60). La première phase, jusqu'en 1920, vit la formation du marché et le début de sa réglementation. Le marché n'en étant qu’à ses premiers pas, les directives émises par le gouvernement général de Taiwan (Taiwan sōtoku-fu 臺灣總督府) occupèrent, dans un premier temps, une place dominante : en effet, le gouvernement général contrôlait

* Université Nihon 日本大学. 
le contenu de la publicité et de la programmation. Cette surveillance se relâcha ensuite à mesure qu'un marché se constituait par lui-même. La deuxième phase, du milieu des années 1920 au milieu des années 1930, est caractérisée par l'élargissement du marché et par sa diversification : diffusion du cinéma jusque dans l'arrière-pays, introduction du cinéma chinois continental, création des premiers ciné-clubs à l'initiative des Taiwanais. Enfin, la troisième et dernière phase correspond à la période de guerre, de la seconde moitié des années 1930 jusqu'en 1945. Alors qu'au début des années 1930 le contrôle du marché par les autorités s'était grandement relâché, le commencement de la seconde guerre sino-japonaise, en 1937, marqua le retour d'une emprise plus stricte de la part du gouvernement général.

Divers canaux de diffusion du cinéma existaient du fait d'une situation où cohabitaient dominants japonais et dominés taiwanais. Durant la phase pendant laquelle le marché du cinéma connut son plus fort essor, à partir du milieu des années 1920, on ne recense pas moins de huit modalités de diffusion ( $c f$. tableau 1 et Misawa $2010: 40$ ).

\section{TABLEAU 1}

Canaux de diffusion du cinéma à Taiwan (1925-1935)

\begin{tabular}{|c|c|c|c|c|c|c|c|c|}
\hline Lucratif/ & \multicolumn{6}{|c|}{ Activité lucrative } & \multicolumn{2}{|c|}{ Activité non lucrative } \\
\hline $\begin{array}{l}\text { Type de } \\
\text { projection }\end{array}$ & \multicolumn{2}{|l|}{\begin{tabular}{|l|} 
Projection \\
itinérante
\end{tabular}} & \multicolumn{4}{|c|}{ Bâtiment dédié } & \multicolumn{2}{|c|}{ Projection itinérante } \\
\hline $\begin{array}{l}\text { Organisateur } \\
\text { et } \\
\text { gestionnaire }\end{array}$ & Taiwanais & Japonais & Taiwanais & & Japonais & & $\begin{array}{l}\text { Taiwanais } \\
\text { (associa- } \\
\text { tions cultu- } \\
\text { relles, etc.) }\end{array}$ & \begin{tabular}{|l} 
Japonais \\
(gouv. \\
général)
\end{tabular} \\
\hline $\begin{array}{l}\text { Langue } \\
\text { utilisée }\end{array}$ & taiwanais & japonais & taiwanais & japonais & japonais & taiwanais & taiwanais & japonais \\
\hline Public visé & Taiwanais & $\begin{array}{l}\text { Taiwanais } \\
\text { Japonais }\end{array}$ & Taiwanais & $\begin{array}{l}\text { Taiwanais } \\
\text { Japonais }\end{array}$ & \begin{tabular}{|l|} 
Taiwanais \\
Japonais
\end{tabular} & Taiwanais & Taiwanais & Japonais \\
\hline & $a$ & $b$ & c & d & e & $\mathrm{f}$ & $g$ & $\mathrm{~h}$ \\
\hline
\end{tabular}

Tableau établi par l'auteure 
Des tentatives de production cinématographique par des Taiwanais virent le jour, même si, d'ordre encore très expérimental, elles n'eurent pas de lendemain commercial. Autant qu'on le sache, sur le demi-siècle d'existence du Taiwan colonisé, le nombre de films produits par des Taiwanais demeura très faible. Taiwan connut le cinéma essentiellement au travers des films japonais réalisés en métropole - les productions japonaises locales, par exemple à des fins de propagande, furent rares -, et secondairement des films hollywoodiens ou chinois. Par exemple pour 1930 : $78 \%$ des films autorisés par le gouvernement général étaient japonais, $10 \%$ américains, $10 \%$ chinois continentaux et $2 \%$ européens.

À titre de comparaison, la Chine, pourtant fragmentée à la même époque, possédait sa propre production de longs métrages, dont la plaque tournante était Shanghai. Cette production, très vigoureuse, vit sa croissance multipliée par 20 de 1923 à 1926¹. Le nombre de salles gérées par des Chinois suivit également cette dynamique ${ }^{2}$. En outre, dans la première moitié des années 1930, le marché du film chinois, qui pouvait s’appuyer sur la diaspora, étendait son influence à l'étranger, y compris Taiwan, même si les films continentaux y furent peu nombreux, tandis que de grandes compagnies étaient désormais établies ${ }^{3}$.

En Corée colonisée, dont le contexte général est plus proche de celui de Taiwan, plus d'une cinquantaine de compagnies cinématographiques virent le jour des années 1920 aux années 1930. En 1941, sur les dix studios en activité, plus de la moitié étaient dirigés par des Coréens. En comptabilisant même les productions « tendancieuses " de gauche et les plus nationalistes,

1. Ainsi furent produits 5 films en 1923, 16 films en 1924, 51 films en 1925, 101 films en 1926. Données tirées de Zhongguo dianying zong mulu 中国电影总目录 (Catalogue général des films chinois), fascicule 1, fac-similé au Zhongguo dianying ziliaoguan 中国 电影資料馆, 1960 .

2. Sur 250 salles existant en Chine continentale dans les années 1930, entre 50 et 60 étaient consacrées aux films chinois (Zhai Min 翟民, “Guopian fuxing yundong zhong guonei yingyuan zhuangkuang zhi yiban» 國片復興運動中國内影院状況之一斑 [Un aspect de la situation des cinémas au sein du mouvement pour le renouveau du cinéma national], Yingxi zazhi 影戲雜誌 [Revue du cinéma], n 718, 1930 ; cité par Hong Shi 1996 : 209-211).

3. Parmi les compagnies pionnières du marché extérieur chinois, on peut évoquer la Tianyi yingpian gongsi 天一影片公司 (Hong Yawen 2000 : 25). 
ce sont près de 200 films coréens qui furent produits sur toute la période coloniale, employant plus d'une dizaine de milliers de personnes ${ }^{4}$.

Autant dire que Taiwan fait figure de parent pauvre des investissements en capitaux "autochtones" au sein de la production cinématographique des colonisés de l'empire japonais. Pour expliquer la mobilisation de tels capitaux en Chine - non colonisée - et en Corée colonisée, on a pu faire état d'un fort soutien du public au cinéma local, lequel reposait sur un certain nationalisme ${ }^{5}$. On pouvait ainsi lire, en 1943, dans la revue Eiga shunpō 映畫旬報, qu’il «suffi[sait] qu'un film soit coréen pour que le public [de la péninsule] vienne inconditionnellement assister aux projections " (Chōsen eiga bunka kenkyūjo 1943 : 18). Ce comportement est à saisir, en ces temps de développement de l'industrie du cinéma, dans un contexte général où les productions étrangères, aussi bien en Europe qu'en Asie, étaient considérées comme autant d'invasions de la sphère culturelle nationale.

Comment Taiwan vient-il se situer par rapport à ces tendances générales, alors que son marché du cinéma prend son envol et se diversifie ? C'est en partant de cette problématique que la présente contribution souhaite aborder la question du nationalisme à Taiwan en contexte colonial (nationalisme des dominés, mais également des dominants) et de ses rapports avec la réception réservée au cinéma. Nous verrons que c'est l'appréhension des particularités constitutives de l'île et de ses habitants qui permet de comprendre un contexte dans lequel le secteur cinématographique ne s'industrialise pas, contrairement à ce qui se passe dans d'autres territoires de l'empire colonial.

La question de la réception des œuvres cinématographiques, essentiellement japonaises, par les populations taiwanaises se pose avec acuité du fait de la situation coloniale. Comme nous l'avons souligné dans notre ouvrage

4. En 1939, l'organisation qui structure l'activité cinématographique des Coréens, l'Association professionnelle du cinéma coréen (Chosŏn yŏnghwa-in hyŏphoe 朝鮮映 畫人協會), était déjà en place (Chōsen eiga bunka kenkyūjo 1943 : 99-114, Ho 2001 : 46-94, Yi 1986).

5. Sur la Chine, se reporter à Xiao (1997). Xiao explique la politique de censure du gouvernement de Nankin vis-à-vis du cinéma, dans la décennie 1927-1937, par le rejet de l'impérialisme occidental et japonais, ainsi que par l'émergence d'une conscience nationale. La présence renforcée de leurs ennemis communistes dans les studios et dans les milieux artistiques en général motiva également cette politique. 
sur l'histoire du cinéma à Taiwan (Misawa 2010 : 22-24), la construction du champ cinématographique au début du $\mathrm{xx}^{\mathrm{e}}$ siècle en Europe, aux ÉtatsUnis et au Japon, en tant que représentation culturelle de la "nation", ne correspond pas terme à terme à l'expérience qu'a connue le Taiwan colonisé - expérience elle-même différente de celle de la Corée. Le cinéma devint un enjeu en Allemagne à la veille de la Première Guerre mondiale (Hansen 1990) et les productions étrangères furent peu à peu rejetées comme émanant d'une altérité devant être différenciée du soi constituant la communauté imaginée. Un processus similaire a pu être observé aux États-Unis vers 1907-1909 lorsque Pathé, qui était en position de force jusque-là, fut peu à peu écarté du marché national (Abel 1995). Cependant, comme nous tenterons de le montrer, une autre culture que celle du cinéma des Étatsnations a été créée à Taiwan durant la période coloniale, culture qui n’a pas rejeté l'altérité - c'est-à-dire les Japonais - mais qui l'a intégrée au travers d'un processus original que nous appelons «assimilation par confrontation " (rinjōteki dochakuka 臨場的土着化).

La théorie de la réception de Hans Robert Jauss, qui a insisté sur le rôle actif du lecteur dans l'histoire des œuvres littéraires (Jauss 1970), ou celle de la transaction de Louise Rosenblatt (Rosenblatt 1978) qui, au-delà de l'interaction entre le lecteur et l'œuvre, a souligné l'influence de la situation et de l'environnement sur les œuvres littéraires, ont guidé notre approche. Lidée d'assimilation par confrontation nous semble permettre de réfléchir sur le cinéma comme événement ou topos dont les Taiwanais firent l'expérience durant la période coloniale.

Après avoir rapidement exposé les cadres du nationalisme et ce que nous entendons par " taiwanais " dans une première partie, nous décrirons la façon dont le cinéma s'est développé dans l'île, ainsi que les politiques du gouvernement général à ce sujet. Puis, dans un troisième temps, nous analyserons l'idée selon laquelle le cinéma japonais aurait été rejeté par la population durant l'époque coloniale, en distinguant les cas des Chinois taiwanais et des aborigènes. Dans un quatrième temps, nous discuterons de l'idée de boycott, qui est au centre de l'historiographie du Guomindang. Enfin, nous verrons comment le concept d'assimilation par confrontation permet d'appréhender la réception du cinéma japonais à Taiwan sous domination coloniale. 


\section{Le nationalisme à Taiwan sous domination coloniale}

Tout d'abord, qu'il nous soit permis d'expliquer brièvement ce que nous entendons ici par nationalisme à Taiwan sous domination coloniale. Par "Taiwanais", nous désignons les populations nées et ayant grandi dans l'île, de langue et de culture chinoise (descendants d'une première colonisation de l'île par les populations du Fujian, à partir du XvII siècle), à distinguer des résidents coloniaux ${ }^{6}$. Nous prenons toutefois bien soin de ne pas considérer ces populations "chinoises " comme les représentantes exclusives des populations de l'île. En effet, Taiwan est une société pluriethnique qui comprend des Chinois d'origines très différentes et dans laquelle il faut aussi compter les aborigènes, davantage mis en avant depuis la démocratisation dans les années $1990^{7}$. C'est pour des raisons de limites

6. Après la chute de l'empire colonial japonais en 1945, cette opposition entre « insulaires " (hontōmin 本島民) et " métropolitains " (naichijin 内地人) se transforma en une animosité entre Chinois " continentaux » (waishengren 外省人) et "locaux " (benshengren 本省人; ceux-là mêmes qui étaient appelés "insulaires " par les Japonais durant l'époque coloniale), comme devait le montrer la répression par le Parti nationaliste lors des événements du 28 février 1947. Ce problème sera désigné du terme de shengji maodun 省籍矛盾 (tensions entre régions d'origine), qui rend compte d'une société dans laquelle les populations chinoises continentales minoritaires, suivant les forces de la République de Chine déchues, se retrouvaient au pouvoir, alors que les populations locales en étaient exclues. Depuis 1990, il est désormais question de si da zuqun 四大族群 (quatre grands groupes ethniques) pour désigner la population de l'île : les Holo, les Hakka, les nouveaux habitants (les anciens " continentaux »), les natifs (anciens « insulaires "). Indépendamment de l'époque d'arrivée de leurs ancêtres dans l'île, tous ces habitants sont ceux que l'on appelle les «Taiwanais ", et les continentaux sont les nouveaux Taiwanais (Wakabayashi 2001).

7. Wakabayashi Masahiro explique qu'une société organisée autour des colons chinois s'était déjà clairement constituée à l'époque de la dynastie Qing et qu'en plus d'une séparation nette avec les populations aborigènes de cultures différentes, des limites avaient été instituées entre sous-groupes au sein des populations chinoises elles-mêmes (Chinois du Zhangzhou, du Quanzhou, mais également les Hakka). C'est sur les fondations de cette société pluriethnique de l'époque des Qing que, sous la domination coloniale japonaise, s'est instauré un système ajoutant une distinction entre "insulaires " (Taiwanais) et «barbares » (les aborigènes). Il s'est ainsi formé une société dans laquelle la conscience d'appartenir à une ethnie demeure forte. Dans ce cadre, les catégories d' "insulaires » ou de "Taiwanais » dépassent celle de Holo (du Fujian) ou de Hakka (davantage du nord de la Chine), "insulaires " étant une catégorisation instituée d'en haut par les maîtres 
de la documentation que les «Taiwanais » de notre enquête sont ainsi surtout ceux de culture chinoise. Toutefois, dans la mesure du possible, nous évoquerons également le cas des populations aborigènes.

Par ailleurs, le nationalisme auquel nous faisons référence est celui des dominants, c'est à dire des Japonais dans le cas de Taiwan. Il correspond au nationalisme dont Benedict Anderson a analysé l'émergence et les processus de reproduction en Russie, dans l'Empire britannique ou au Japon. Il s'agit de la conscience de peuple qui se dégage à l'issue de mouvements populaires engagés au XIX ${ }^{\mathrm{e}}$ siècle, selon des modalités interchangeables d'un pays à l'autre. Derrière ce qu'il appelle " nationalismes officiels ", Anderson estime qu'il se cache presque toujours un fort décalage entre nation et empire (Anderson 2002 : chap. 5).

Dans le cas japonais, l'historien Komagome Takeshi souligne que l'idée d'assimilation - avancée de manière à faire état d'une intégration culturelle positive - dissimulait en réalité la généralisation et la reproduction d'un système de ségrégation à plusieurs niveaux (Komagome 1996: 357). Il opère une distinction entre deux usages du nationalisme : le "nationalisme linguistique " et le "nationalisme du sang", le premier ayant une fonction englobante, là où le second instaure une mécanique de l'exclusion ${ }^{8}$. La question de l'assimilation à Taiwan a été étudiée par Chen Peifeng, qui s'est penché sur la réception des politiques d'enseignement du japonais aux habitants de l'île. En établissant deux tendances, celle de l'assimilation à la civilisation, et celle de l'assimilation ethnique, il a pu conclure que

japonais de l'île, là où "Taiwanais » est une catégorie élaborée par le discours nationaliste antijaponais des populations dominées. Dans le Taiwan actuel, une division en trois niveaux structure désormais la représentation multiculturelle de la société : population aborigène ou chinoise ? Si chinoise, native de l'île ou arrivée après 1945 (insulaires ou continentaux) ? Si insulaire, Holo ou Hakka ? Cette distinction suit la division en quatre grands groupes évoquée plus haut (Wakabayashi 2008 : 32-39, 336).

8. Komagome insiste sur la nécessité de distinguer deux dimensions au terme d'assimilation : une dimension concrète, relevant des caractéristiques du système de pouvoir, et une dimension abstraite, conceptuelle. Dans la logique du pouvoir, il faut encore distinguer les deux dimensions de l'égalité et de la discrimination. Sur le plan du droit, cela se matérialise par la dichotomie entre expansion dans le prolongement de la métropole et domination coloniale ; sur le plan culturel, cela s'incarne dans une dichotomie entre assimilation à soi et pseudo-autonomie concédée aux dominés (Komagome $1996: 20)$. 
le principe d'assimilation avait fonctionné comme la justification d'une logique tant égalitariste que ségrégationniste. Les Taiwanais accueillirent les deux facettes de ce mécanisme de manière sélective (Chen Peifeng 2010 : 294-311).

Le nationalisme des dominés, les Taiwanais, n'était pas non plus sans présenter de complexité. Wakabayashi Masahiro explique que si ce dernier était un "nationalisme antijaponais", illustré par l’idée de la "libération de Taiwan ", il faut néanmoins opérer une distinction entre les "patriotes ", qui étaient pour une unification à la Chine continentale, et les " révolutionnaires " ou "réformateurs insulaires ", partisans de l'autonomie, c'est-à-dire de l'indépendance de l'île (Wakabayashi 1985 : 110-131). Cependant, tous se retrouvaient essentiellement autour de l'idée de la libération du joug japonais.

Le cadre des nationalismes de l'île une fois posé, penchons-nous sur notre sujet : la question de la réception du cinéma étranger à Taiwan et le soutien dont bénéficièrent les productions locales du temps de la domination coloniale japonaise, durant la première moitié $\mathrm{du} \mathrm{xx}^{\mathrm{e}}$ siècle.

\section{Le gouvernement général de Taiwan face au succès du cinéma chinois}

Le soutien économique apporté aux productions cinématographiques locales et le boycott des productions étrangères procèdent tous deux d'un processus dans lequel le cinéma est considéré comme une forme d'expression de la culture nationale. Il est désormais établi que cette forme de nationalisme précède la Première Guerre mondiale. Mais c'est surtout avec l'omniprésence de l'industrie cinématographique américaine, dans la seconde moitié des années 1920, que l’on voit émerger, en Allemagne, en Grande-Bretagne et en France, des politiques de quota limitant la diffusion des productions américaines sur les marchés nationaux ${ }^{9}$. La Grande Guerre avait fait prendre conscience de l'importance du cinéma en tant qu'outil destiné à édifier et à influencer les masses et chaque pays développait ses

9. Rappelons brièvement que l'Allemagne mit en place un système limitant l'importation de films étrangers en 1925, qui fut suivi par une loi britannique en 1927, puis par une limitation à l'importation des films américains en France en 1928. 
propres productions (Kittler 1986). La même prise de conscience eut lieu au Japon peu après.

Les documents conservés aux archives diplomatiques renferment de précieux indices à ce sujet : ici, un article de journal à l'attention du ministre des Affaires étrangères, informant de la puissance de l'influence du cinéma aux États-Unis ${ }^{10}$, là une demande de prêt - émise par un diplomate - d'un film présentant le Japon ${ }^{11}$, ou encore une demande de renforcement de la politique de contrôle des productions japonaises susceptibles d'être projetées à l'étranger, afin d'être certain de ne pas donner une mauvaise image du pays ${ }^{12}$. Le marché lui-même est politiquement impliqué puisque, par exemple, au rejet des immigrants japonais aux États-Unis répond le rejet, au Japon, des productions cinématographiques américaines (Tanaka 1924). Il est donc tout à fait clair qu'à l'époque du Japon colonial, le cinéma, industrie de divertissement, était considéré comme un support culturel national, tant par le gouvernement que par le public.

D'importants changements de direction marquèrent la politique coloniale japonaise après la Première Guerre mondiale, dans le Taiwan du début des années 1920 (Haruyama 1980 : 48). Ils reflétaient une conception des territoires coloniaux « dans le prolongement de la métropole », au sein d'un contexte plus large d'appel à l'autodétermination des peuples depuis la fin de la guerre. Cette nouvelle situation eut des répercussions sur le cinéma : à partir du milieu des années 1920, les diffusions de films chinois et les

10. Note envoyée par le consul de New York en poste, Aneha Junpei 姉歯準平 (?-?), à l'attention du ministre des Affaires étrangères Uchida Kōsai 内田康哉 (1865-1936), «Beikoku ni okeru katsudō shashin ryūkō chizu zukai sōfu no ken » 米國ニ於ケル活動 寫眞流行地圖圖解送附/件 (Schéma explicatif de la diffusion et de l'influence du cinéma aux États-Unis d'Amérique), daté du 23 juillet 1923, archives du ministère des Affaires étrangères (I.1.12.0.1).

11. “Gaimushō shoyū firumu taiyo kata ni kansuru ken " 外務省所有フイルム貸與方 二關スル件 (Modalités de prêt des films en possession du ministère des Affaires étrangères), 27 juillet 1926, archives du ministère des Affaires étrangères (A.3.3.0.2-2).

12. Note de l'envoyé extraordinaire et ministre plénipotentiaire en Suisse, Ariyoshi Akira 有吉明 (1876-1937), à l'attention du ministre des Affaires étrangères Shidehara Kijūrō 幣原喜重郎 (1872-1951), "Nihon no fūshū ni kansuru katsudō shashin “firumu” ni kansuru ken" 日本/風習二關スル活動寫眞「フィルム」 二關スル件 (Au sujet des " films » traitant des mœurs japonaises), archives du ministère des Affaires étrangères (I.1.12.0.1). 
projections à but non commercial organisées par les Taiwanais furent soumises à une réglementation (décret $n^{\circ} 58$ de 1926 du gouvernement général de Taiwan) sensiblement identique à celle en vigueur en métropole.

En conséquence de cette interaction entre le contexte politique et l'importance reconnue aux productions cinématographiques, les films chinois (i.e. continentaux), a-t-on souligné, furent l'objet d'une censure sévère de la part du gouvernement général de Taiwan, qui mit finalement en place à leur encontre une très stricte interdiction d'importation en 1937, lors du déclenchement de la seconde guerre sino-japonaise (Lü 1961 : 24, Ye 1996 : 94, Ye 1998 : 284). Cette mesure ne semble pourtant pas avoir été systématique, même après 1937 . Néanmoins, les importations furent progressivement limitées et, dans certaines régions, il fut complètement interdit de les diffuser et de les projeter durant la période 1937-1945. Quant aux limitations antérieures, comme le montre le tableau 2, le contrôle des productions chinoises était déjà draconien dans l'entre-deux-guerres. Le rapport entre le nombre de films chinois touchés par des coupes et une moyenne fixée à 1 est de 2,5 pour l'année 1927. Ce rapport est de 3,1 en 1928 , pour descendre à 1,2 en 1929 , puis remonter à 2,2 films en $1930^{13}$. La sévérité de la censure des autorités japonaises vis-à-vis du cinéma chinois en particulier est ainsi bien avérée.

TABLEAU 2

Taux de censure par provenance des films (1927-1930)

\begin{tabular}{|l|r|r|r|r|}
\hline & films japonais & films chinois & films américains & films européens \\
\hline 1927 & 0,15 & 2,46 & 0,44 & 0,95 \\
\hline 1928 & 0,09 & 3,09 & 0,49 & 0,33 \\
\hline 1929 & 0,93 & 1,2 & 0,88 & 0,99 \\
\hline 1930 & 0,79 & 2,23 & 0,49 & 0,49 \\
\hline
\end{tabular}

Tableau établi par l'auteure à partir des données figurant dans Taiwan sōtoku-fu keimu-kyoku (1931)

13. En prenant l'année 1927 pour indice 1, la moyenne des volumes de coupe dans les films diminue à 0,84 en 1928 , pour ensuite augmenter à 1,92 en 1929 , et jusqu'à 3,4 en 1930. Le volume de censure cinématographique augmente ainsi de façon générale, audelà des films chinois. En 1936, la police expliquait encore, dans une de ses publications, que « les censeurs cinématographiques, conformément à l'idée de [colonies qui représentent le] prolongement de la métropole, font preuve d'impartialité » (Nomura 1936 : 148-150). 
À juger de la sévérité de la censure et des limitations dont firent l'objet les films chinois, on comprend clairement que ce sont bien ces derniers, pourtant relativement moins nombreux que les productions occidentales, qui inquiétaient déjà les autorités avant le déclenchement de la guerre du Pacifique. La popularité du cinéma chinois à Taiwan était perçue par le colonisateur comme un phénomène propre à l'île (Taiwan sōtoku-fu keimu-kyoku 1931 : 229-230). Le gouvernement général avait conscience de la sympathie éprouvée par le public taiwanais à l'endroit de la Chine, pays antagoniste au colonisateur japonais, et considérait cette situation comme naturelle dans la mesure où 4 millions d'insulaires avaient culturellement beaucoup en commun avec leurs voisins continentaux (ibid.). Le cinéma chinois venait défier, à Taiwan, le pouvoir colonial et son nationalisme officiel. Pour preuve, le tonnerre d'applaudissements réservé, en 1928, à la projection d'un film sur le mariage de Tchang Kaï-chek et Song Meiling. Le journal Taiwan minbao 臺灣民報 souligna à l'époque " qu'on ne pouvait que constater la force de la sympathie des Taiwanais à l'égard des Chinois » et que « les différences de psychologie des peuples conduisent tout naturellement à des divergences dans les centres d'intérêt et de divertissement. Ces différences dans les goûts cinématographiques démontrent ainsi amplement l'impossibilité des buts poursuivis par les politiques d'assimilation» (Taiwan minbao 1928). Lors de la projection de ce film, il devait également y avoir dans le public des personnes illettrées qui purent partager par l'image les réjouissances de leur "patrie» (et de ses habitants, les Chinois), créant par là-même une occasion d'imaginer ensemble ce qu'était cette dernière. L'historien Lü Sushang rapporte l'événement suivant.

En 1928, parmi les films chinois apportés dans l'île par Ke Ziqi 柯子岐 de la compagnie Qiming 啓明 de Xiamen, dont Le dieu de la paix [Heping zhi shen 和平之神] ou La rose de la renaissance [Fuhuo de meigui 復活的 玫瑰], se trouvait un documentaire intitulé Chronique de la conquête du Nord par Tchang Kaï-chek [Chiang Jieshi beifa ji 蒋介石北伐記], qui fut l'objet de projections organisées avec Wu Jingyang 只鯨洋 de Xinzhu (c'est-à-dire Wu Yousan 只幼三 qui travaille actuellement au cinéma Wanguo Xiyuan 万國戯院 à Taipei). Pour l'occasion, Wu loua la salle du Yongle Xiyuan 永樂戯院, et le succès populaire fut tel que, craignant que la familiarité de Taiwan avec la Chine ne stimule trop le patriotisme des masses, le pouvoir japonais fit couper la scène du film dans laquelle apparaissait le Père de la nation [Sun Yatsen 孫逸仙]. (Lü 1961 : 18) 
Comme nous le verrons plus loin, le cinéma chinois était fréquemment lié à l'opposition nationaliste contre la colonisation japonaise. Au nom de son nationalisme officiel, le pouvoir colonial se méfiait donc de cet engouement des Taiwanais, qui aurait pu être l'expression d'un sentiment d'appartenance au même peuple. Mais partout, l'idée de "retenue " prévalait. Un fonctionnaire du gouvernement général notait en 1931 : "Éviter de faire des plaisanteries, même amusantes, sur ses beaux-parents devant son épouse relève du sens commun. Et de la même façon, lorsque des populations différentes sont réunies, il faut que chacun fasse preuve de retenue afin d'éviter de provoquer des tensions inter-ethniques" (Takahashi 1931) ${ }^{14}$.

On peut ainsi clairement percevoir chez le colonisateur une attitude et des politiques de défense d'un nationalisme officiel de circonstance. Qu'en était-il d'un éventuel nationalisme insulaire?

\section{Un rejet du cinéma japonais en contexte colonial à Taiwan ?}

Les recherches menées après 1945 et la décolonisation ${ }^{15}$ ont fréquemment conclu qu'en raison du patriotisme taiwanais (pro-continental), le cinéma chinois avait été chaleureusement accueilli et que les productions japonaises avaient été ignorées (Lü 1961 : 20, Chen Guofu 1985 [1984]). La popularité du cinéma chinois et le traitement particulier qu'il reçut des autorités coloniales à Taiwan sont confirmés par le tableau 3, qui met en rapport la censure subie par les films en fonction de leur provenance, entre 1926 et 1935. Les importations de films chinois baissèrent fortement après l'incident de Mandchourie en 1931, avant de remonter l'année suivante aux $10 \%$ occupés précédemment. Certains ont même pu rapporter qu' «avant l'incident, ce qui frappait dans la progression du cinéma chinois à Taiwan, c'est que

14. Le bureau de l'Information du Premier secrétaire du gouvernement général (Taiwan sōtoku-fu kanbō jōhō-ka 臺灣總督府官房情報課) était en charge de la revue Taiwan jihōo 臺灣時報, qui était publiée en interne.

15. Il est malaisé de saisir l'après août 1945 comme un " après-guerre " dans le cas de Taiwan, comme l'a souligné Liu Jinqing 劉進慶 (Liu 2006), puisque la guerre civile continuait. Nous préférons ici évoquer un "après 1945 " afin de renvoyer à l'aprèsSeconde Guerre mondiale et à la défaite japonaise. 
presque tous les films chinois étaient importés» (Ichikawa 1941 : 94). Il est probable qu'il y ait eu une attitude de rejet voire de boycott du cinéma japonais par les insulaires, ainsi que le suggèrent des interviews menées auprès de Chen Yongsheng 陳勇㙫, ancien bonimenteur cinématographique de langue taiwanaise ${ }^{16}$.

Pourtant, il convient de prendre les conclusions de ces travaux avec prudence, dans la mesure où ils furent tous réalisés à l'époque du gouvernement dictatorial d'après 1945, dirigé par les nationalistes du Guomindang qui venaient de fuir le continent et s'étaient emparés du pouvoir après les Japonais. À l'heure où il convenait surtout de mettre en avant le "patriotisme " des Taiwanais, il était délicat d'évoquer les spécificités historiques et culturelles de l'île, ainsi que les effets de la colonisation japonaise sur ces dernières. Mais selon la classification de Wakabayashi Masahiro évoquée plus haut, deux mouvances s'opposaient dans le nationalisme antijaponais à Taiwan, concernant le sens à donner à une libération de l'île : une qui prônait le "rattachement à la patrie " (la Chine) et une autre, "révolutionnaire", qui revendiquait son autonomie (Wakabayashi 1985 : 110-131). Puisqu'il est question de nationalisme culturel, il est important de se demander si les mêmes clivages n'auraient pas existé dans la réception du cinéma. Autrement dit, il faudrait déterminer si d'autres attitudes n'auraient pas existé à côté d'un rejet "patriotique " du cinéma japonais. De fait, la société taiwanaise, dans sa pluralité, ne présentait pas partout les mêmes facilités d'accès au cinéma chinois. Une attitude de rejet pur et simple du cinéma japonais n'était pas toujours envisageable.

D'une part, les importations de films chinois ne commencent qu'au milieu des années 1920, période avant laquelle il était donc impossible d'y avoir accès. D'autre part, après l'incident de Mandchourie, dans un contexte où l'importation des films était limitée, très peu de productions chinoises étaient susceptibles de circuler dans l'île. Et même si les copies

16. Entretiens réalisés en notre présence les 11 et 12 décembre 1998 au domicile de Chen Yongsheng par une équipe du Guojia dianying ziliaoguan 國家電影資料館 avec Hong Yawen 洪雅文, Xue Huiling 薛恵玲 et Wang Meiling 王美㱓. Chen est un chinois taiwanais, né en 1932 puis élevé à Zhanghua. Il avait été lié au milieu du cinéma dès son jeune âge, par son père qui dirigeait la compagnie Guanghua yingye gongsi 光華影業 公司 et son oncle qui était bonimenteur - c'est-à-dire conteur pour le cinéma muet -, métier qu'il exerça à son tour dès l'adolescence. 
pouvaient circuler et être diffusées en relative abondance, le public ayant accès aux projections payantes était limité à celui des classes moyennes et supérieures des grandes villes. Pour le public des classes les plus pauvres ou vivant loin des grands centres urbains, les seules occasions d'aller au cinéma se limitaient aux projections itinérantes gratuites.

Le cas des aborigènes taiwanais, confinés dans des zones administratives spéciales - appelées "zones barbares » (banchi $i$ 蕃地) - et objets d'une politique de ségrégation, est sans aucun doute l'exemple le plus clair de populations qui n'avaient pas accès au cinéma commercial. Les professionnels du cinéma et leurs circuits ne pouvaient inclure ces "zones barbares", où les seuls films disponibles étaient ceux des projections du gouvernement général. Par exemple, lors de la projection organisée en 1926 par l'Association de la police de Taiwan (Taiwan keisatsu kyōkai 臺灣警察協會), la majorité des spectateurs n'avait encore jamais vu de film. La police rapporte le "succès immense et indiscutable " de l'événement, et indique que le public s'y pressait (Taiwan keisatsu kyōkai zasshi 1926). Dix bobines furent projetées à cette occasion, dont De la conquête du monde animal par l'homme (Dōbutsu seifuku eiga 動物征服映畫) et Reportage sur la visite de Son Altesse le Prince Impérial dans notre île (Kötaishi denka hontō gyōkei no jikkyō 皇太子殿 下本島行啓の實況). En outre, les projections de films sur les déplacements du prince étaient accompagnées d'exposés sur le système impérial, lors desquels «les organisateurs se levaient et retiraient leur couvre-chef dès que des images de Son Altesse apparaissaient " à l'écran, afin d'encourager le public à rendre ce type de salut. Les aborigènes qui avaient appris la langue japonaise « se chargeraient ainsi de transmettre à leur tour ces explications à leurs frères et sœurs, à leurs parents ", pour montrer « la grandeur de l'effort éducatif " déployé par le Japon, et faire comprendre à leur entourage que la maitrise du japonais leur permettrait de profiter de nombreux nouveaux loisirs.

He Jiming 何基明, responsable après 1937 de «l'éducation par le cinéma " au sein de la section "éducation " du bureau des Affaires intérieures de la province de Taichū (Taizhong), rapporte que les films montrés par son bureau lors de projections itinérantes, organisées selon un axe nordsud, deux fois l'an dans près de quarante hameaux aborigènes, "avaient des contenus relevant surtout de l'actualité du moment». On montrait les nouvelles cinématographiques durant vingt à trente minutes, par exemple à propos de "la chute de Nankin ou de la chute de Xuzhou ", ainsi qu'un 
court métrage, l'ensemble étant suivi d'une discussion durant laquelle les organisateurs expliquaient que le Japon « est actuellement en guerre contre la Chine. Vous devez travailler sérieusement et soutenir du mieux possible l'effort productif " (Yang Yifeng 1994: 64) ${ }^{17}$. Ce type de programme relevait clairement de la propagande, mais les villageois se réjouissaient pourtant des projections dès qu' elles étaient annoncées et, nous dit-on, "venaient dès le matin sur la place [du hameau] pour attendre le début du film en fumant des cigarettes".

Comment les spectateurs perçurent-ils les films projetés dans ce type d'espace ? Iwali Piho, née en 1926 et déplacée à l'âge de six ans vers le village de Sakura à Nantou ${ }^{18}$, explique avoir vu, quand elle était enfant, quatre ou cinq projections de "films militaires" par an, dans la cour de l'école publique japonaise. Les films muets étaient commentés par un bonimenteur, mais il était difficile de l'entendre à cause du bruit ambiant. Comme "les occasions de voir des films étaient rares" et comme "les projections étaient gratuites ", les habitants du hameau se rassemblaient pour y assister et « remplissaient le terrain de sport ", rapporte-t-elle ${ }^{19}$.

Il est possible d'évoquer d'autres exemples concernant les populations chinoises des plaines. Li Maosong 李茂松, originaire de Jiayi, se souvient avoir assisté, lorsqu'il était enfant, à une ou deux projections par an dans la cour de l'école publique. Il explique : «Les gens s'habillaient avec de beaux vêtements, comme pour le jour de l'An, et on accrochait des lanternes en papier depuis l'entrée de l'école. C'était vraiment comme une fête, tout cela pour voir ces images animées. Quel moment de joie quand on était petit $^{20}$ !»

17. Nous nous appuyons ici sur une interview de He Jiming réalisée le 3 juin 1993 par une équipe composée de Yagi Nobutada 八木信忠, Ikeda Hiroshi 池田博, Karasuyama Masaharu 烏山正晴, Watanabe Yutaka 渡辺豊, Hirosawa Fuminori 広沢文則, Maruyama Hiroshi 丸山博 et Yamana Izumi 山名泉. Cette interview est conservée par le Guojia dianying ziliaoguan, et elle a été traduite en chinois après le décès de He Jiming (Yang Yifeng 1994).

18. Originaire du village de Tau, Iwali Piho fait partie de l'ethnie Atayal. Elle porte le nom chinois Pan Laihao 潘来好 et le nom japonais Nakayama Yuriko 中山百合子.

19. Interview d'Iwali Piho, menée à son domicile par l'auteure, le 28 mars 1999.

20. Interview de Li Maosong, menée par l'auteure, à Yulanzhuang (Taipei) le 2 juin 1998. 
Outre les projections du gouvernement général, Zhang Mingde 張明德 (Chinois originaire de Taizhong, né en 1926) se souvient de projections organisées au titre de loisirs dans les locaux d'usines sucrières. Il rapporte : "La ségrégation était terrible entre Japonais et insulaires. On plaçait un tapis, ou bien un tatami ou un coussin de sol à l'endroit le mieux situé, au milieu de la salle. C'était la place réservée aux Japonais, tandis que les ouvriers insulaires s'asseyaient sur des chaises disposées derrière. Quant à nous, on lorgnait par la fenêtre. C'était la première fois que je voyais un film !»

Qu'elles soient organisées par le gouvernement général ou par des usines japonaises, ces projections différaient de celles des salles de cinéma permanentes des villes. Les descriptions et souvenirs rapportés ci-dessus font clairement comprendre que l'espace même dans lequel ces films non commerciaux étaient regardés était un lieu de propagande mis en place par le pouvoir colonial. Malgré tout, pour les spectateurs, le film demeurait attrayant en soi, en tant que "spectacle", et non pour son contenu narratif. À ce sujet, Iwali Piho apporte d'ailleurs une précision : "Nous regardions simplement les gens à l'écran, nous ne comprenions pas ce qui était raconté ». Selon Li Maosong, le film attirait pour la "fête » qui accompagnait la projection. Ainsi ce n'est pas parce que la diffusion du cinéma subissait un puissant contrôle que le public était privé de sa subjectivité dans la réception des films. Il y avait aussi une dimension ludique : le cinéma pouvait être considéré comme un loisir, se déroulant dans un espace autorisant une grande pluralité d'interprétations et de discussions. D'ailleurs, comme nous le verrons dans la partie suivante, même l'Association pour la culture de Taiwan (Taiwan wenhua xiehui 臺灣文化協會, fondée en 1921 à Taipei par Jiang Weishui 蔣渭水 (1891-1931)), dont la ligne était bien plus « antijaponaise " que les établissements assurant des projections commerciales, avait diffusé à ses débuts des films japonais ${ }^{21}$. Le tableau 3 indique qu'une majorité des films américains et japonais était approuvée par le bureau de

21. Voir le journal Taiwan minbao (1926: 101). L'association acheta ensuite des bobines à Shanghai. "Tokuyō shisatsujin no kōdō ni kansuru ken, Taiwan bunka kyōkai, Sai Baika " 特要視察人)行動二關スル件 臺灣文化協會 蔡培火 (Cai Peihuo de l'Association pour la culture de Taiwan, personne devant être l'objet d'une surveillance particulière), in Futeidan kankei zakken. Taiwanjin no bu 不遈團關係雜件 臺灣人/部 (Affaires diverses sur les groupes insoumis : les Taiwanais), archives du ministère des 
la Censure, ce qui permet de comprendre qu'ils étaient avant tout perçus comme non-problématiques, comme du divertissement en somme. Le fait que les films chinois aient bénéficié d'un bon accueil ne signifiait donc pas mécaniquement que les films japonais aient été boycottés.

\section{TABLEAU 3}

Films approuvés par le bureau de la Censure cinématographique du gouvernement général de Taiwan, en pourcentage relatif par pays d'origine

\begin{tabular}{|r|r|r|r|}
\hline & productions japonaises & productions américaines & productions chinoises \\
\hline 1926 & 70 & & \\
\hline 1927 & 70 & & 4 \\
\hline 1928 & 68 & 28 & 4 \\
\hline 1929 & 80 & & 10 \\
\hline 1930 & 78 & & 5 \\
\hline 1931 & 83 & 10 & 10 \\
\hline 1932 & 78 & 15 & 8 \\
\hline 1933 & 75 & 19 & 9 \\
\hline 1934 & 70 & 18 & 6 \\
\hline 1935 & 63 & & \\
\hline
\end{tabular}

Tableau établi par l'auteure à partir des chiffres fournis par Ide (1942). Ceux-ci sont approximatifs : quoique peu nombreux, des films européens sont également entrés à Taiwan, mais ces données ne les mentionnent pas.

\section{Une historiographie en débat}

Si l'existence d'un public prenant plaisir aux projections organisées par le pouvoir colonial est avérée, au-delà, nous devons souligner que les réactions de l'audience étaient structurées par le système hiérarchique qui organisait le Taiwan colonisé. Les populations des secteurs situés en dehors des circuits de diffusion du cinéma commercial, illettrées ou pas assez fortunées pour payer leur billet, furent les plus touchées par la politique déployée par le gouvernement général. De fait, si les spectateurs profitaient de

Affaires étrangères (4.3.2.2-2). Nous remercions Chen Wensong 陳文松 d'avoir attiré notre attention sur ces documents. 
l'ambiance festive et ludique des projections, et étaient surtout intéressés par le prodige de ces "images qui bougent", ces dernières et les explications données durant les projections n'en légitimaient pas moins le système colonial dont elles faisaient la propagande. L'acte même de participer à ces événements intégrait le spectateur au système colonial. C'est cette situation qui, après 1945, allait conditionner la mise en place d'un discours chez le Guomindang incriminant les Taiwanais, "population aliénée ». De là s'est construite la vision affirmant que l'époque se caractérisait par un «bon accueil fait aux films chinois » et un "boycott des films japonais », vision qui a occupé une place conséquente dans l'historiographie du cinéma à Taiwan après 1945.

On comprend bien sûr qu'un tel discours a pu s'imposer chez les historiens dans le Taiwan d'après 1945, compte tenu de la proximité avec la période coloniale. Pourtant, l'impossibilité de démontrer l'existence d'un boycott systématique des productions cinématographiques japonaises, alors que le patriotisme chinois antijaponais est bien avéré, pourrait plutôt constituer l'une des caractéristiques de la réception du cinéma à Taiwan sous domination coloniale, et non une anomalie dont il faudrait s'étonner.

Nous pouvons envisager trois types de configurations pour comprendre cette réception.

Dans la première, la population aurait estimé que Taiwan relevait de l'État-nation chinois. Dans ce cas, le «bon accueil fait au cinéma chinois " et le "boycott des films japonais" iraient de pair, comme l'a soutenu le discours dominant en histoire du cinéma depuis 1945.

Dans la deuxième, les Taiwanais auraient considéré leur île comme relevant de l'État-nation japonais. Aucun nationalisme antijaponais n'aurait alors existé, en une configuration inverse à celle de l'hypothèse précédente, avec cette fois un "bon accueil fait aux films japonais " et un "boycott des films chinois ». Cette position serait en conformité avec le nationalisme officiel de l'époque en faveur de la métropole japonaise.

Dans la troisième, les Taiwanais se seraient considérés comme un Étatnation à part entière. On observerait alors un « soutien aux films taiwanais » et un «boycott des films chinois et japonais".

Étant donné qu'un nationalisme antijaponais a de fait existé, la deuxième configuration ne peut être pleinement considérée. La première et la troisième, en suivant les catégories de nationalismes proposées par Wakabayashi Masahiro, relèvent de l'ordre du possible. Toutefois, aucune de ces deux 
hypothèses, ni celle d'un «boycott des films japonais ", ni celle d'un «boycott de tous les films étrangers, chinois et japonais ", ne peut être clairement vérifiée. On ne peut en outre postuler l'existence d'un quelconque « soutien aux films taiwanais " étant donné la rareté de ces derniers. La situation est donc différente.

\section{Une assimilation par confrontation au travers du commentaire en taiwanais}

Il faut, pour mieux comprendre l'histoire du cinéma dans l'île, se pencher sur une des modalités d'assimilation ou de "taiwanisation" des films qui circulaient par les canaux proprement taiwanais. On sait que toutes les projections pour le public taiwanais mobilisaient le talent d'un bonimenteur de langue taiwanaise (voir le tableau 1, colonnes a, c, f, g). Cette pratique, qui avait débuté avec les films muets, s'était poursuivie pour les films parlants. Le rôle du bonimenteur était de raconter, de commenter et d'expliquer le contenu du film au public dans une langue que celui-ci comprenait. Un tel personnage était certes nécessaire pour les films japonais ou occidentaux, ceux-ci ne bénéficiant que des sous-titres en japonais, mais aussi pour les films chinois, car la langue de Taiwan (langue du Fujian) différait du mandarin utilisé dans les productions de Shanghai. En outre, l'équipement nécessaire au cinéma parlant était encore peu répandu dans les années 1930, à l'exception des salles permanentes des grandes villes. Or, si le nombre de bonimenteurs en métropole chuta rapidement dans la seconde moitié des années 1930 avec l'avènement du cinéma parlant, le nombre de leurs homologues taiwanais resta, quant à lui, stable (tableau 4$)^{22}$. Ils ne se contentaient

22. Nous avons également identifié, de façon partielle, pour l'année 1933 à Taichū 臺中 (Taizhong), neuf bonimenteurs métropolitains et dix «insulaires " c'est-à-dire taiwanais. Pour l'année 1934 dans la province de Karen 花蓮 (Hualian), deux métropolitains et aucun insulaire. Pour l'année 1934 dans la province de Tainan 臺南 (Tainan), vingt bonimenteurs, mais nous n'avons pas de données ethniques sur ceux-là. Néanmoins, dans tous ces cas, y compris pour Taipei, il ne s'agit que de personnes ayant un permis officiel. Or, il semble que de nombreux bonimenteurs aient opéré sans avoir de permis (interview de Chen Yongsheng). Voir Taichū-shū keimu-bu (1933), Karen-kō chō keimu-ka (1934), Tainan-shū keimu-bu (1935). 
pas de traduire les dialogues ou les sous-titres, mais en profitaient parfois pour discuter de politique avec le public, en improvisant au passage des caricatures satiriques. Ainsi, les commentaires du bonimenteur modifiaient profondément le contenu des images projetées. Alors que les films taiwanais n'existaient pour ainsi dire presque pas, et qu'il eut été impossible de produire un contenu local, une "taiwanisation " par confrontation se produisait donc dans la rencontre entre ces films et la société taiwanaise, via le bonimenteur.

TABLEAU 4

Nombre des bonimenteurs cinématographiques pour la province de Taihoku (Taipei), années 1931-1941

\begin{tabular}{|l|r|r|}
\hline & Japonais & Taiwanais \\
\hline 1930 & 20 & 10 \\
\hline 1931 & 30 & 15 \\
\hline 1932 & 28 & 25 \\
\hline 1933 & 32 & 26 \\
\hline 1934 & 29 & 23 \\
\hline 1935 & 29 & 35 \\
\hline 1936 & 32 & 27 \\
\hline 1937 & 12 & 31 \\
\hline 1938 & 13 & 32 \\
\hline 1939 & 6 & 29 \\
\hline 1940 & 5 & 22 \\
\hline 1941 & 5 & 24 \\
\hline
\end{tabular}

Tableau établi par l'auteure à partir de Taihoku-shū chiji kanbō bunsho-ka (19321942), partie « Keisatsu torishimari ni zoku suru shokugyō oyobi dantai » 警察取締二 屬スル職業及團體 (Professions et organisations devant être surveillées par la police)

Les films étrangers et notamment japonais n'étaient donc pas boycottés dans ces circuits de diffusion proprement taiwanais, qui étaient pourtant plus proches du nationalisme antijaponais. Ils étaient assimilés et « taiwanisés " par confrontation et hybridation avec la langue locale. Nous utilisons cette formulation d' "assimilation par confrontation" pour distinguer ce processus de celui de "créolisation" (Misawa 2004). On parle en effet de langue créole dans le cas où un pidgin (langue commune utilisée entre des personnes de langues différentes) s'est stabilisé pour devenir la langue 
principale d'une population donnée, l'idée de "créolisation » renvoyant donc au processus qui voit l'établissement d'une telle langue hybride. Mais l'assimilation par confrontation ne concerne qu'un espace et un moment donnés. D'où l'importance capitale de ces bonimenteurs.

Alors que la diffusion d'une même pellicule cinématographique aurait dû garantir un contenu chaque fois fidèle et identique, la confrontation des images avec les propos du bonimenteur en transformait le sens. Ainsi le lieu même du visionnage conférait une coloration particulière au film. Lidée d'assimilation par confrontation, ainsi formulée, ne désigne donc pas un phénomène propre aux situations coloniales ou à l'histoire du cinéma. Au-delà, elle pourra plus généralement servir à analyser des phénomènes relevant de l'interculturalité. Le cinéma comme art reproductible est, historiquement, dépendant du niveau des connaissances techniques et du système de production. La place de la performance individuelle, et donc de la " malléabilité " par confrontation, recule au fur et à mesure que la technologie progresse. Pourtant, dans le cas de Taiwan, les bonimenteurs de langue taiwanaise continuèrent d'être actifs non seulement après l'avènement du film parlant (à la différence du Japon), mais encore après 1945. Ce dernier point s'explique par le fait que si les films chinois produits sur le continent, en mandarin, proposaient un contenu culturel théoriquement plus proche des us et coutumes de l'île, ils restaient en réalité difficiles à saisir pour le public taiwanais.

On peut en outre considérer l'assimilation par confrontation, sur ces lieux de loisir, comme l'effet d'une revendication nationale chez ces populations qui ne manifestaient pas d'attitude antijaponaise claire que ce soit en paroles ou en actes. C'est précisément pour cette raison que le gouvernement général essaya de contrôler cette " taiwanisation " constatée au travers de la réception du cinéma, en instaurant un système de "permis " pour les bonimenteurs ${ }^{23}$, ainsi qu'en censurant les fascicules de commentaires prévus pour les films ${ }^{24}$. Malgré cette méfiance, le gouvernement général fut,

23. Décret provincial de Taihoku (Taipei) n² 23, d’octobre 1926, «Kōgyō kisoku » 興行 規則 (Règlement concernant les spectacles). Voir Taihoku-shū keimu-bu (1931 : 587-599). 24. Décret du gouvernement général de Taiwan $n^{\circ} 59$, de juillet 1926, "Katsudō shashin firumu ken.etsu kisoku " 活動寫眞フィルム檢閲規則 (Règlement sur la censure cinématographique) ; ordonnance $n^{\circ}$ 93, de décembre 1939, "Katsudō shashin firumu ken.etsu kisoku toriatsukai kitei » 活動寫眞フィルム檢閲規則取扱規定 (Décision relative 
lui aussi, bien obligé d'avoir recours aux commentateurs lorsqu'il essaya de japoniser les populations taiwanaises. Il ne pouvait en effet se passer de la puissance de ce média visuel qu'est le cinéma pour toucher les foules illettrées.

Il convient de souligner que si le taiwanais fut interdit dans les journaux et les écoles après le déclenchement de la seconde guerre sino-japonaise en $1937^{25}$, il resta néanmoins autorisé pour les projections cinématographiques itinérantes, afin que leur contenu soit bien compris par le public. Dans le même temps, les sous-titres en chinois étaient interdits ${ }^{26}$, sous prétexte que "les personnes souhaitant voir des films n'avaient qu’à apprendre le japonais » (Sugiyama 1943). En 1937, les rubriques de littérature chinoise et de composition en chinois classique furent interdites dans les journaux, tandis que les cours de chinois furent supprimés dans les écoles. On proposa alors de se dispenser des bonimenteurs en taiwanais dans le cinéma (Taiwan

au Règlement sur la censure cinématographique) (Taiwan sōtoku-fu keimu-kyoku 1942).

25. Si les colonnes en chinois des quotidiens furent interdites, des revues intégralement en chinois telles Fengyuebao 風月報 ou Nanfang 南方 furent cependant toujours publiées, même durant la période de guerre (Yang Yongbin 2001). Li Chengji a montré qu'il y eut des programmes de radio permanents, tel le programme musical «Taiwan ongaku " 臺灣音樂 (Taiwan Musique), bien avant que ne soit mis en place le programme d'informations de l'Association radiophonique de Taiwan. Cela s'explique notamment par l'augmentation rapide des auditeurs (Li Chengji 2004 : 287-295).

26. Selon Katō Atsuko, "même un film tel Aizen katsura 愛染かつら [Les camélias de l'amour] fut apprécié par le public taiwanais " (Katō 2003 : 224). Elle indique que ce film fut diffusé avec des sous-titres chinois sous le titre Airan chun 愛染椿, mais ne précise pas les lieux de projection. Elle note également que « les films japonais furent présentés tels quels, ou bien avec de légers sous-titres de soutien, et étaient visionnés par le public quasiment de la même façon qu'en métropole, sans être particulièrement préparés pour l'audience locale " (ibid. : 228). Katō s'appuie ici sur l'étude de Tamura (2000), qui indique qu'Aizen katsura avait été projeté à Shantou, sur le continent, et que cette information avait été rapportée par un Japonais, selon lequel « environ un tiers du public était alors composé de Chinois » (Tamura 2000 : 223-224). Concernant les sous-titres, l'ouvrage de Tamura se contente de supposer que « les Taiwanais, qui sont des entrepreneurs habiles et motivés, étaient sans doute capables d'insérer rapidement [...] des caractères écrits sur un transparent disposé devant la pellicule " (ibid. : 237). Étant donné l'écart entre la conclusion de Katō et le propos de Tamura, sur laquelle s'appuyait pourtant cette première, il est difficile de se prononcer avec certitude sur l'effective diffusion de films japonais avec sous-titres chinois à Taiwan. 
kōron 1937). Mais, finalement, les autorités coloniales considérèrent «ne pas pouvoir se passer des commentaires en langue taiwanaise, compte tenu de l'état actuel de la diffusion de notre langue nationale " (Taiwan geijutsu shinpo 1938). Dans les faits, l'utilisation de bonimenteurs en taiwanais perdura jusqu’à la décolonisation japonaise en $1945^{27}$. Voici, à ce sujet, ce que rapporte Chen Yongsheng, qui effectuait ce travail en langue taiwanaise $e^{28}$.

Les cinémas pour les Japonais, c'étaient les salles principales du Yanping Xiyuan 延平戯院 à Tainan ou du Jinjiguan 金雞館 à Gaoxiong. Il n'y avait que des Japonais là-bas. Pratiquement aucun Taiwanais, à peine quelques-uns de ceux qui avaient eu une éducation japonaise conséquente. Mais il y avait toujours un bonimenteur dans les autres salles, la deuxième ou la troisième, dans lesquelles se rendaient massivement les Taiwanais. Même dans les grandes villes : quand il y avait un commentaire, c'était toujours pour le taiwanais. Les Japonais comprenaient les sous-titres ou bien regardaient des films japonais parlants. Mais la plupart des Taiwanais ne comprenaient rien au japonais. Même après le début de [la politique assimilatrice d'] « impérialisation » [à partir de 1937], les gens étaient trop occupés à gérer leurs affaires quotidiennes et personne ne parlait couramment japonais. Et puis, nous, nous utilisions le taiwanais pour «transmettre la culture japonaise » auprès de la population, pour qu'elle comprenne, sans quoi cela ne sert à rien, non ? Obliger à utiliser le japonais n'aurait provoqué que de l'hostilité, et c'est pour cela que, moi aussi, je suis resté bonimenteur en taiwanais jusqu'à la fin de la guerre. Nous n'avons jamais été obligés d'utiliser prioritairement le japonais, que ce soit pour les films récents ou pour les [anciens] films retouchés. Si le public ne comprend pas ce qui est raconté, ça n'a aucun sens, hein ?

Benedict Anderson notait à propos de la domination coloniale : «À la longue, la seule réponse au secret qui entoure la langue des opprimés est la retraite, ou d'autres massacres" (Anderson 2002 : 152). Nous pouvons justement constater, dans le cas précis des projections cinématographiques à Taiwan, que l'idée d'assimilation, c'est-à-dire une japonisation conforme au nationalisme officiel du colonisateur, dut battre en retraite quand elle se heurta à ce « secret » qu'était le parler des colonisés.

27. Par exemple, l'Association pour l'édification par le cinéma pour la province de Taihoku organisa un événement à l'occasion du "Jour pour le réconfort des burakumin" en mai 1940. À cette occasion, elle "fit attention à prévoir un bonimenteur de langue taiwanaise pour les films historiques [japonais] Chūshin-gura 忠臣蔵, Nankō fushi 楠公父子, et quelques autres" (Ōsaka mainichi shinbun Taiwan-ban 1940).

28. Interview de Chen Yongsheng (voir note 16). 


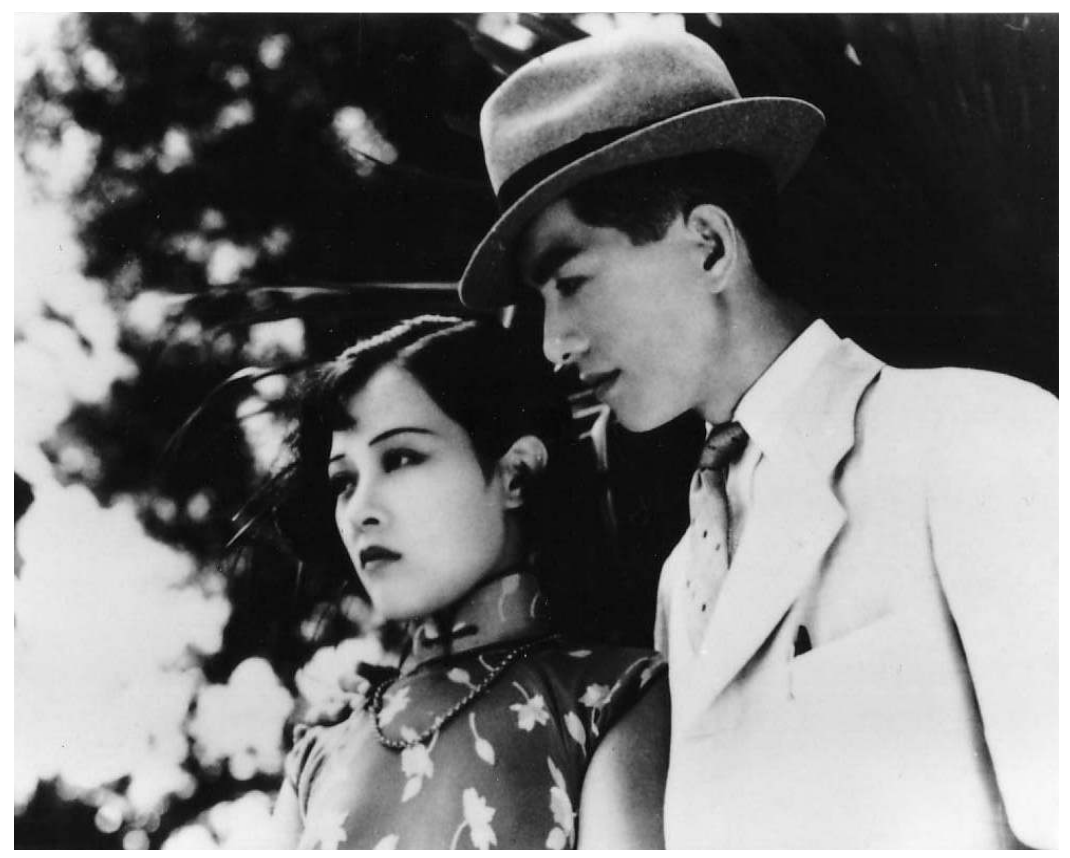




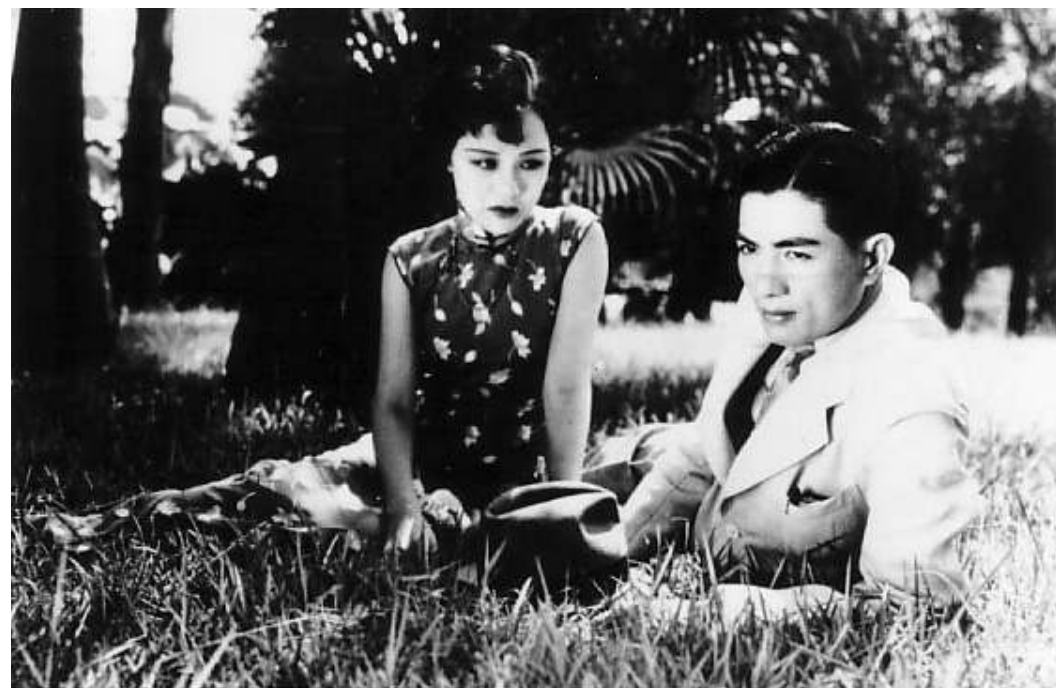

Fig. 1 \& 2. Photographies publicitaires pour le film Bōshunfū 望春風 (1937, produit par Wu Xiyang 呉錫洋), I'une des rares productions taiwanaises de l'époque coloniale. Une version sonore fut enregistrée, mais elle fut doublée en japonais afin de mettre en avant l'unité avec la métropole, comme le voulait l'idéologie du temps de la guerre de 1937-1945. Cette version doublée fut l'objet de critiques par le public taiwanais, qui se plaignait de «ne rien ressentir si la langue taiwanaise n'est pas utilisée ». Voir le courrier « Eiga-hyō Bōshunfū C.K.B. » 映畫評『望春風』(Mon avis sur le film Bōshunfū, par C.K.B.), revue Taiwan fujin-kai 臺灣 婦人界 (Le monde de la femme taiwanaise), février 1938, p. 79. Ces photographies appartiennent à l'acteur principal, Peng Kailian 彭楷棟 (dont le nom japonais était Nitta Tōichi 新田棟一), que l'on voit sur la droite. Nous remercions Luo Fuquan 羅福全 et Lin Xiuqin 林秀琴 pour leur aide. 


\section{Conclusion}

Lorsque l'industrie du cinéma se développa à Taiwan des années 1920, le gouvernement général craignit que les productions chinoises continentales ne viennent renforcer la conscience nationale chez les Taiwanais, ce qui aurait conduit à une contestation de la domination coloniale. Nous avons vu que les Taiwanais firent, de fait, un bon accueil aux films chinois. Ces éléments concordent avec les résultats de la recherche menée en histoire du cinéma à Taiwan après 1945. Celle-ci estima en substance que, durant la période coloniale, les Taiwanais réservèrent un bon accueil aux films chinois "du fait de leur patriotisme » et qu'ils boycottèrent les films japonais "par esprit de résistance». Nous avons cependant montré que les Taiwanais n'avaient pas toujours eu accès aux productions chinoises, et qu'ils n'avaient pas nécessairement toujours boycotté les films japonais. Dans certains cas, les dominés se divertirent même avec les programmes de propagande du dominant.

Les commentaires des bonimenteurs, personnages d'importance capitale pour le divertissement cinématographique à Taiwan, permettent, nous semble-t-il, de comprendre pourquoi à un «bon accueil des films chinois" ne correspondit pas un "boycott des films japonais". Les films étrangers, y compris japonais, diffusés par des circuits proprement taiwanais, se voyaient tous "taiwanisés " par un processus d'hybridation impliquant la langue taiwanaise. Nous avons appréhendé cette appropriation des contenus cinématographiques au travers du concept d'" assimilation par confrontation ", qui peut aussi éclairer la façon dont ceux qui ne pouvaient exprimer ouvertement de revendication nationale formulèrent une résistance à la domination coloniale. La persistance d'espaces culturels de langue taiwanaise, y compris dans des lieux où l'on projetait des films de propagande, constitue un élément crucial à prendre en compte dans l'analyse historique de la situation coloniale si l'on considère que les mouvements de résistance armée étaient systématiquement réprimés, ou que les médias de langue chinoise furent interdits durant la guerre de l'Asie et du Pacifique (1937-1945).

Le cinéma parlant de langue taiwanaise apparut dans les années 1950. Les bonimenteurs n'étaient plus utiles dans ce nouveau contexte d'un cinéma considéré comme "réellement taiwanais ». Il n'était dès lors plus nécessaire de "s'approprier» les films étrangers. De façon intéressante, le nouveau 
marché cinématographique taiwanais d'après 1945 produisit divers remakes locaux en taiwanais de films chinois ou japonais à succès. On pourrait voir ici le passage d'un processus d'assimilation par confrontation via un bonimenteur, à un phénomène de "créolisation " impliquant l'élaboration de contenus reproductibles "vernacularisés ". C'est sans doute là un aboutissement nécessaire du processus d' "assimilation " des produits, dérivant du fonctionnement intrinsèque de l'industrie cinématographique qui adapte sans cesse les contenus aux demandes des marchés et des publics. Ce phénomène d'appropriation après 1945 des films japonais dont le contenu était intégralement "taiwanisé ", nous semble constituer une étape du processus de décolonisation du marché cinématographique taiwanais (Misawa 2012).

L'évolution de la situation du cinéma à Taiwan après 1945 serait ainsi une sorte d' " autopoïèse ", les contenus cinématographiques se voyant sans cesse réélaborés en suivant les demandes de la population. Dans ce cadre, l'assimilation par confrontation, nécessaire du temps de la domination coloniale, se transforma en "créolisation" des contenus, qui permettait leur transmission directe au public. L'histoire de la production du cinéma à Taiwan durant l'époque coloniale, du point du vue du capital «autochtone ", paraît extrêmement déséquilibrée. Mais considérer la réception du cinéma modifie cette perspective, puisqu'à défaut de films locaux, les productions étrangères furent réinterprétées et présentées d'une façon qui les enracinait dans l'île, processus qui continua après 1945 sous une autre forme.

Traduit par Arnaud Nanta et Laurent Nespoulous 


\section{Bibliographie}

\author{
ABEL Richard 1995 \\ «The Perils of Pathé, or the \\ Americanization of Early American \\ Cinema », in Leo Charney \& Vanessa R. \\ Schwartz (eds.), Cinema and the \\ Invention of Modern Life, Berkeley, \\ University of California Press : 182-223.
}

\section{ANDERSON Benedict 2002}

L'imaginaire national. Réflexions sur l'origine et l'essor du nationalisme, trad. Pierre-Emmanuel Dauzat, Paris, La Découverte, [Imagined Communities. Reflections on the Origin and Spread of Nationalism, London, Verso, revised and extended edition, 1991].

\section{CHEN Guofu 陳國富 1985 [1984]} « Zhimin yu fan-zhimin / Taiwan zaoqi dianying huodong 》殖民與反殖民 / 臺灣早期電影活動 (Colonialisme et anticolonialisme / Les premiers temps du cinéma à Taiwan), Pianmian zhi yan 片面之言, Taipei, Zhonghua minguo dianying tushuguan chubanbu 中華民國 電影圖書館出版部: 88-92, [1 $1^{\text {re éd. }}$ esous le titre «Zhimin yu fan-zhimin » 殖民與 反殖民, Jinri dianying 今日電影，165， août 1984].

\section{CHEN Peifeng 陳培豊 2010}

" Dōka » no dōshō imu - Nihon tōchika

Taiwan no kokugo kyōiku-shi saikō

「同化」の同床異夢一日本統治下台湾の

国語教育史再考 (Les multiples facettes de I'« assimilation ». Repenser I'histoire de l'enseignement du japonais à Taiwan sous administration japonaise), Tokyo, Sangensha 三元社.

Chōsen eiga bunka kenkyūjo 朝鮮映畫 文化研究所 (dir.) 1943

"Chōsen eiga sanjū shūnen-shi » 朝鮮 映畫三○周年史 (Trente années de cinéma coréen), Eiga shunpō 映畫旬報 (Revue du cinéma), 11 juillet 1943 : 99-114.

\section{HANSEN Miriam 1990}

« Early Cinema: Whose Public

Sphere? ", in Thomas Elsaesser \& Adam Barker (eds.), Early Cinema: Space, Frame, Narrative, London, BFI : 228-245.

HARUYAMA Meitetsu 春山明哲 1980 "Kindai Nihon no shokuminchi tōchi to Hara Kei » 近代日本の植民地統治と原敬 (Hara Kei et la domination coloniale par le Japon moderne), in Haruyama Meitetsu \& Wakabayashi Masahiro 若林正丈 (dir.), Nihon shokuminchi-shugi no seijiteki tenkai 1895-1934. Sono tōchi taisei to Taiwan no minzoku undō 日本植 民地主義の政治的展開1895-1934年 その統治体制と台湾の民族運動 (Le développement du colonialisme japonais de 1895 à 1934 . Son système de domination et les mouvements de revendication nationale à Taiwan), Tokyo, Ajia Seizai Gakkai アジア政経学会.

\section{HO Hyŏnch'an 扈賢贊 2001} Waga shinema no tabi. Kankoku eiga o furikaeru わがシネマの旅 韓国映画を 振りかえる (Un voyage dans notre cinéma. Retour sur le cinéma coréen), trad. Nemoto Rie 根本理恵, Tokyo, Gaifūsha 凱風社. 
HONG Shi 弘石 1996

«Wusheng de cunzai » 无声的存在

(L'existence du muet), in Zhongguo dianying ziliaoguan 中国电影資料馆 (dir.), Zhongguo wusheng dianying 中国无声 电影 (Le cinéma chinois muet), Pékin, Zhongguo dianying chubanshe 中国 电影出版社.

\section{HONG Yawen 洪雅文 2000}

« Zhimin shiqi de Taiwan dianying 18991948 »殖民時期的臺灣電影 (1899-1948) (Le cinéma à Taiwan pendant l'époque coloniale 1899-1948), in Huang Jianye 黄建業 \& Huang Ren 黄仁 (dir.), Shiji huigu: tushuo huayu dianying 世紀回顧:

圖説華語電影 (Souvenirs du siècle. Le cinéma de langue chinoise), Taipei, Wenjianhui 文建會 : 67-69.

\section{ICHIKAWA Sai 市川彩 1941}

Ajia eiga no sōzō oyobi kensetsu アジア 映畫の創造及建設 (Création et édification du cinéma asiatique), Tokyo, Kokusai eiga tsūshin-sha 國際映畫通信社.

IDE Kiwata 井出季和太 1942

Taiwan chisekishi 臺灣治績誌

(Les résultats de notre politique à

Taiwan), Taihoku, Taiwan nichinichi shinpōsha 臺灣日日新報社.

\section{JAUSS Hans Robert 1970}

Literaturgeschichte als Provokation, Frankfurt am Main, Suhrkamp Verlag [trad. japonaise Kutsuwada Osamu 縺田收, Tokyo, Iwanami Shoten 岩波 書店, 1976].

\author{
Karen-kō chō keimu-ka \\ 花蓮港廳警務課 (dir.) 1934 \\ Shōwa 9 nen kankō Karenkō-chō keimu \\ yöran 昭和9年刊行花蓮港廳警務要覽 \\ (Rapport annuel de la police du port de \\ Hualian, pour l'an 9 de l'ère Shōwa).
}

\section{KATŌ Atsuko 加藤厚子 2003}

Sōdōin taisei to eiga 総動員体制と映画

(Le système de mobilisation générale et le cinéma), Tokyo, Shin.yō-sha 新曜社.

\section{KITTLER Friedrich 1986}

Gramophone, Film, Typewriter, trad.

G. Winthrop-Young \& M. Wutz, Stanford, Stanford University Press.

\section{KOMAGOME Takeshi 駒込武 1996}

Shokuminchi teikoku Nihon no bunka tōgō 植民地帝国日本の文化統合

(L'intégration culturelle dans l'empire colonial japonais), Tokyo, Iwanami shoten 岩波書店.

\section{Chengji 李承機 2004 \\ Taiwan kindai media-shi kenkyū josetsu.} Shokuminchi to media 台湾近代メディア史 研究序説: 植民地とメディア (Introduction à l'étude des médias modernes à Taiwan. Médias et colonialisme), thèse de doctorat soutenue à l'université de Tokyo, Faculté des Arts et Lettres.

\section{LIU Jinqing 劉進慶 2006}

" "Sengo" naki higashi Ajia - Taiwan ni ikite»「戦後」なき東アジア·台湾に生きて (Vivre à Taiwan et en Asie de l'Est, où il n'y a pas eu d'« après-guerre »), Zenya 前夜, $9: 229-246$. 
LÜ Sushang 呂訴上 1961

Taiwan dianying xiju shi 臺灣電影

戲劇史 (Histoire des spectacles

cinématographiques à Taiwan), Taipei,

Yinhua chuban 銀華出版.

MISAWA Mamie 三澤真美恵 2004

«Shokuminchi-ki Taiwan ni okeru eiga fukyū no "bunsetsu-teki keiro" to “konsei-teki dochaku-ka"» 植民地期 台湾における映画普及の<分節的経路 $>$ と <混成的土着化> (La diffusion du cinéma dans le Taiwan de l'époque coloniale. Segmentation et enracinement par hybridation), Ritsumeikan gengo bunka kenkyū 立命館言語文化研究 (Revue d'étude des langues et civilisations de I'université Ritsumeikan), XV-3 : 39-52.

\section{MISAWA Mamie 2010}

«Teikoku» to « sokoku » no hazama. Shokuminchiki Taiwan eiga-jin no kōshō to ekkyō「帝国」と「祖国」のはざま一 植民地期台湾映画人の交渉と越境 (Entre « empire » et « patrie ». Négociations et transgressions des gens du cinéma taiwanais à l'époque coloniale), Tokyo, Iwanami shoten 岩波書店.

\section{MISAWA Mamie 2012}

«Aliénation ou acculturation coloniale? Taiwan et "l'énigme" d'un succès : le Festival du film japonais de Taipei (1960) », trad. Arnaud Nanta, Laurent Nespoulous \& Anne Kerlan, Cipango, 19: 13-54.

NOMURA Kōichi 野村幸一 1936 «Keimu-kyoku eiga ken.etsu-shitsu haiken »警務局映畫檢閲室拝見 (En visite à la salle de censure cinématographique de la police), Taiwan keisatsu jihō 臺灣 警察時報 (Nouvelles de la police de Taiwan), janvier 1936, 242 : 148-150.

Ōsaka Mainichi shinbun Taiwan-ban 大阪毎日新聞臺灣版 (journal Ōsaka mainichi, édition pour Taiwan) 1940 «Burakumin no i.an-bi. Kitai sareru kyōka eiga no katsudō. Nōson no katsudō-ryoku o yashinau » 部落民の 慰安日 期待される教化映畫の活動 農村の活動力を培ふ (Le jour pour le réconfort des burakumin. Les films de I'Association pour l'édification sont attendus. Renforcer l'activité des villages), 15 mai 1940.

\section{ROSENBLATT Louise M. 1978}

The Reader, the Text, the Poem: the Transactional Theory of the Literary Work, Carbondale, Southern Illinois University Press.

\section{SUGIYAMA Shizuo 杉山静夫 1943}

《Taiwan eiga-kai bekken » 臺灣映畫界 瞥見 (Aperçu du monde du cinéma à Taiwan), Eiga shunpō 映畫旬報, 21 novembre 1943 : 272-273.

Taichū-shū keimu-bu 臺中州警務部 (dir.) 1933

Shōwa 8 nen kankō. Taichū-shū keimu yōran 昭和8年刊行 臺中州警務要覽 (Rapport annuel de la Police de la province de Taizhong, pour l'an 8 de l'ère Shōwa), Taichū, gouvernement général de Taiwan. 
Taihoku-shū chiji kanbō bunsho-ka 臺北州知事官房文書課 (Bureau des archives du premier secrétaire du préfet de la province de Taipei) (dir.) 1932-1942

Taihoku-shū tōkei sho 臺北州統計書 (Documents statistiques pour la province de Taipei), années 1931 à 1941, 12 vol., Taihoku, gouvernement général de Taiwan.

Taihoku-shū keimu-bu 臺北州

警務部 (dir.) 1931

Taihoku-shū keisatsu hōki (jō) 臺北州 警察法規 (上) (Règlements policiers pour la province de Taipei, vol. 1), Taihoku, gouvernement général de Taiwan.

Tainan-shū keimu-bu 臺南州警務部 (dir.) 1935

Shōwa 9 nenmatsu genzai. Tainan-shū keimu yōran 昭和9年末現在 臺南州警務 要覽 (Rapport annuel de la police de la province de Tainan, pour la fin de l'an 9 de l'ère Shōwa), Tainan, gouvernement général de Taiwan.

\section{Taiwan geijutsu shinpō 臺灣㙯術新報 1938}

«Shakai kyōka to eiga katsuyō. Hontōjin taishū ni tai suru eiga no motsu jūdai shimei » 社會教化と映畫活用 本島人大衆に 對する映畫のもつ重大使命 (De l'utilisation du cinéma pour l'édification de la société. La grande mission du cinéma à l'intention des masses insulaires), $1^{\text {er }}$ août $1938: 1$.
Taiwan keisatsu kyōkai zasshi 臺灣警察 協會雜誌 (Revue de l'Association de la police de Taiwan) 1926

Compte-rendu de projections itinérantes, 104 : 242-244.

\section{Taiwan kōron 臺灣公論 1937}

«Kaisetsusha taiwango haishi » 解説者 臺灣語廢止 (L'abolition de la langue taiwanaise pour les commentateurs), 5 décembre $1937: 29$.

\section{Taiwan minbao 臺灣民報 1926}

«Wenxie huodong xiezhenbu chushi » 文協活動寫眞部出世 (La naissance de la section cinéma de l'Association pour la culture de Taiwan), 18 avril 1926, 101 : 7.

\section{Taiwan minbao 1928}

«Zhongguo yingxi dahaoping JiangSong jiehun de renqi »中國影戲大好評 蒋宋結婚的人気 (Grand succès pour le film chinois sur le mariage du couple Tchang Kaï-chek), 13 mai 1928, 208 : 7.

Taiwan sōtoku-fu keimu-kyoku 臺灣 總督府警務局 (Département de la police du gouvernement général de Taiwan) (dir.) 1931

Taiwan no keisatsu 臺灣の警察 (La police de Taiwan), Taihoku, gouvernement général de Taiwan.

Taiwan sōtoku-fu keimu-kyoku 臺灣 總督府警務局 (Département de la police du gouvernement général de Taiwan) (dir.) 1942

Taiwan keisatsu hōki (ge) 臺灣警察法規

(下) (Règlements de la police de Taiwan, dernier vol.), Taihoku, gouvernement général de Taiwan. 
TAKAHASHI Sotojirō 高橋外次郎 1931

«Taiwan ni okeru eiga ken.etsu »

臺灣に於ける映畫檢閲 (La censure cinématographique à Taiwan), Taiwan

jihō 臺灣時報, septembre 1931 : 11-14.

TAMURA Shizue 田村志津枝 2000

Hajime ni eiga ga atta. Shokuminchi

Taiwan to Nihon はじめに映画があった

植民地台湾と日本 (Et le cinéma fut. Le

Taiwan colonisé et le Japon), Tokyo,

Chūō kōron-sha 中央公論新社.

\section{TANAKA Saburō 田中三郎 1924}

«Beikoku eiga haiseki mondai hitokoto » 米國映畫排斥問題一言 (Du problème du boycott du cinéma américain), Kinema shunpōキネマ旬報, 21 juin 1924, $163: 7$.

\section{WAKABAYASHI Masahiro 若林正丈 1985}

Kaikyō Taiwan seiji e no shiza 海峡 台湾 政治への視座 (Un détroit. Regard sur la politique à Taiwan), Tokyo, Kenbun shuppan 研文出版.

\section{WAKABAYASHI Masahiro 2001}

Taiwan. Henyō shi chūcho suru aidentiti 台湾 変容し躊躇するアイデンティティ (Taiwan. Des identités mouvantes et hésitantes), Tokyo, Chikuma shobō 筑摩書房.

\section{WAKABAYASHI Masahiro 2008}

Taiwan no seiji. Chūka minkoku taiwanka no sengoshi 台湾の政治 中華民国台湾化 の戦後史 (La politique à Taiwan. Histoire de l'après-guerre dans la République de Chine à Taiwan), Tokyo, Tōkyō Daigaku shuppankai 東京大学出版会.

\section{XIAO Zhiwei 1997}

«Anti-Imperialism and Film Censorship During the Nanjing Decade, 19271937 », in Sheldon Hsiao-peng Lu (ed.) Transnational Chinese Cinemas: Identity, Nationhood, Gender, Honolulu, University of Hawai'i Press : 35-57.

\section{YANG Yifeng 楊一峯 1994}

«Dianying-kuang, bashi zai. He Jiming fangtan-lu» 電影狂、八十載 何基明 訪談録 (Propos de He Jiming, un inconditionnel de cinéma, à ses quatrevingt ans), Dianying xinshang 電影欣賞, $70: 52-83$.

YANG Yongbin 楊永彬 2001

«Cong Fengyue dao Nanfang. Xilun yifen zhanzhengqi de zhongwen wenyi zazhi 》従『風月』到『南方』析論一份 戰爭期的中文文藝雜誌 (De Fengyue à Nanfang. Analyse des revues littéraires de langue chinoise durant la période de guerre), in Kawahara Isao 河原功, Guo Yijun 郭怡君 \& Yang Yongbin (dir.), Fengyue, Fengyuebao, Nanfang, Nanfang shiji. Zongmulu, zhuanlun, zhuzhe suoyin 風月·風月報・南方・南方詩集 總目録·專 論·著者索引 (Index général, par thèmes et auteurs, de Fengyue, Fengyuebao, Nanfang, et des poèmes de Nanfang), Taipei, Nantian shuju 南天書局 : 68-150.

\section{YE Longyan 葉龍彦 1996}

Xinzhu shi dianying shi 新竹市電影史 (Histoire du cinéma dans la ville de Xinzhu), Xinzhu, Xinzhu shili wenhua zhongxin 新竹市立文化中心.

\section{YE Longyan 1998}

Ri zhi shiqi Taiwan dianying shi 日治時期 
臺灣電影史 (Histoire du cinéma à Taiwan sous administration japonaise), Taipei, Yushan-she 玉山社.

YI Yŏng-il 李英一 1986

« Nittei shokuminchi jidai no Chōsen eiga »日帝植民地時代の朝鮮映画 (Le cinéma coréen à l'époque de la colonisation japonaise), trad. par Takasaki Sōji 高崎宗司, in Imamura Shōhei 今村昌平, Satō Tadao 佐藤忠男, Shindō Kaneto 新藤兼人, Tsurumi Shunsuke 鶴見俊輔 \& Yamada Yōji 山田 洋次 (dir.), Kōza Nihon eiga 講座日本映画 (Le cinéma japonais), Tokyo, Iwanami shoten 岩波書店, vol 3. : 312-335. 
